Original research

\title{
A randomised controlled feasibility trial of the Graded Repetitive Arm Strengthening Programme (GRASP) delivered to stroke survivors at home
}

\author{
Wilson Dodzo ${ }^{1}$ \\ Robert Grant ${ }^{2}$ \\ Leigh Forsyth ${ }^{3}$ \\ Gita M Ramdharry ${ }^{4}$ \\ ${ }^{1}$ Imperial College Healthcare NHS Trust, St Mary's Hospital, London, UK \\ ${ }^{2}$ Social Care and Education, Kingston University and St George's University of London, London, UK \\ ${ }^{3}$ Leadership \& Quality Improvement Team, NHS Improvement, London, UK \\ ${ }^{4}$ Queen Square Centre for Neuromuscular Diseases, University College Hospitals NHS Foundation \\ Trust, London, UK
}

Correspondence to: Gita M Ramdharry (g.ramdharry@ucl.ac.uk)

Received: 26 May 2017; accepted following double-blind peer review: 18 November 2019

\section{Key words}

Community rehabilitation, GRASP, Stroke, Upper limb

\section{Introduction}

Stroke is a common, serious health care problem and a leading cause of acquired adult disability (Alawieh et al. 2018).. Many people who have had a stroke (stroke survivors) are left with moderate to severe disabilities and dependent on others for daily activities (Saka et al, 2009). About $40 \%$ are left with functional impairments and attendant burden to individuals and societies (Young and Forster, 2007). The Stroke Association (2018), estimates that stroke results in $£ 1.7$ billion in health and social care costs. Reducing this burden requires, in part, optimising stroke rehabilitation (Young and Forster, 2007).

Up to $70 \%$ of stroke survivors report loss of upper limb function after stroke (Wu et al, 2011a). The persistence of upper limb impairments, such as weakness, pain and loss of range of motion, contributes to significant ongoing disability and challenges participation (Ward et al, 2019). The evidence for efficacy of different rehabilitation methods remains limited (Khan et al, 2011), but increased treatment intensity using repetitive task-oriented methods improves motor and functional recovery (Barreca et al, 2003). There has been a recent call for focusing on upper limb rehabilitation with consideration of dosage of input and meaningful movements that can be replicated in function (Ward et al, 2019). Evidence is building for the effectiveness of intensive inpatient upper limb rehabilitation (Ward et al, 2018) but these programmes are short in duration and have strict entrance criteria. There is a place for evidence-based upper limb rehabilitation in the community to promote independence and meaningful engagement with a person's rehabilitation. The advantage here is a more direct transfer into daily activities.

Graded Repetitive Arm Strengthening Programme (GRASP) has mainly been delivered and studied in the inpatient setting, with one small feasibility study of a telephone monitoring model 
(Simpson et al, 2017). GRASP is a self-administered intervention designed to target upper limb function, delivered as a complement to regular therapy (Harris et al, 2009; Eng et al, 2012).

Treatment is supervised by the therapist and not meant to replace direct therapy given by a qualified therapist. GRASP brings together different effective interventions, while also taking advantage of proven principles of motor and neurological recovery (Langhorne et al, 2011). GRASP combines theoretical approaches: functional strengthening, range of motion, weight bearing, trunk control, repetitive paretic upper limb practice, self-management and bilateral upper limb training (Harris et al, 2009). People with upper limb impairment are coached and supported by therapists to engage and progress exercises. It allows compensation and skill acquisition through relearning and repetitive practice (Kollen et al, 2009) and there is evidence for it preventing reduced function through disuse (Eng et al, 2012).

It is acknowledged that community-dwelling patients receive less intensive therapy compared to inpatients due to access to therapists and support (Bernhardt et al, 2008). This could be detrimental to recovery, so a self-managed supplement to therapy, such as GRASP, could increase total therapy time.

Harris et al (2009) conducted a multi-site randomised controlled trial (RCT) to determine the effectiveness of GRASP on upper limb recovery after stroke in an inpatient setting. There were greater improvements in upper limb function in the GRASP group compared to the control group. Significant differences were also reported in favour of GRASP for grip strength and paretic upper limb use. Harris et al (2009) concluded that GRASP was a safe, time-efficient, effective and costeffective method to improve upper limb recovery after stroke Kumar and Kumar (2016) also reported GRASP as effective in improving upper limb function in activities of daily living in acute stroke patients. Another GRASP qualitative study showed that participants felt the involvement of caregivers helped improve upper limb function (Harris et al, 2009). Some issues around fidelity have been raised, however, with how therapists implement GRASP (Connell et al, 2014).

These results cannot be generalised to community care, where, among other considerations, treatment frequency and duration, barriers to participation, psychosocial factors and environment are different. Simpson et al (2017) conducted a small study of an 8-week GRASP programme at home where six participants' progress was monitored by telephone. Improvements were observed with this model of GRASP. In the UK, stroke survivors commonly access community rehabilitation from multidisciplinary teams in their homes. This can be instead of, or following, inpatient rehabilitation.

This study had the following aims:

1) Explore the feasibility of recruitment and engagement in self-administered GRASP for people with stroke receiving community rehabilitation

2) Estimate the effect of self-administered GRASP treatment on functional recovery of the upper limb, using the Wolf Motor Function Test.

\section{Methods}

This study was a prospective, single-blind, parallel group, randomised controlled feasibility trial. Three comparable community rehabilitation teams based in London, UK participated in the study. Occupational therapists, physiotherapists and rehabilitation assistants delivered treatment to participants in their homes. All therapists were trained in GRASP and briefed on the study protocol.

\section{Ethical approval}

Ethical approval was given by the National Research Ethics Service [REC reference number: 12/LO/0676. Written informed consent was obtained from all participants. 
Participants were recruited by convenience sampling from a cohort of patients referred to the community stroke rehabilitation service. Referrals from a 5 month period were screened, and eligible patients were invited to participate.

A total of 10 eligible stroke survivors took part in the study (Table 1). A total of 70 referrals were screened over 5 months for eligibility into the study, with 20 meeting the inclusion criteria. Four potential participants declined to participate and five were excluded for receiving other forms of treatment which could potentially influence the study, so $73 \%(11 / 15)$ of invited, eligible patients agreed to participate. The participants were randomly allocated to GRASP $(n=6)$ or control $(n=5)$ treatment. One participant in the GRASP group refused any further community rehabilitation and so withdrew from the study, yielding a 9\% (1/11) drop-out before post-treatment outcome measures (Figure 1). The recruitment rate over 5 months was 2 participants per month, with a $14 \%$ recruitment of patients screened.

The inclusion criteria were: presence of active scapula elevation, palpable wrist extension, 20 or more on the Folstein Mini Mental Status Examination (Folstein et al, 1975; Eng et al, 2012). Exclusion criteria were: previous stroke diagnosis,neurological comorbidities, premorbid upper limb musculoskeletal problems, and complicating unstable cardiovascular or pulmonary history

Written informed consent was obtained from all participants before being assigned to either the experimental or control group using permuted block randomisation, with each block including random allocation to promote balance between the two groups. All participants received standard conventional rehabilitation of exercises to promote balance and mobility in the home environment (Langhorne et al, 2011). The experimental group also had standardised visits to the participant's home to demonstrate and review GRASP. The study was single-blinded: group allocation was concealed from the investigators.

Table 1. Demographics and clinical characteristics of sample

\begin{tabular}{|l|l|l|}
\hline VARIABLE & GRASP & CONTROL \\
$(n=5)$ & $(n=5)$ & \\
\hline Sex & 4 Female, 1 Male & \\
\hline Age (mean) years & 58 (Range: 30-83) & 62 (Range: 52-77) \\
\hline Dominant arm affected, $n$ & 1 participant & 1 participant \\
\hline $\begin{array}{l}\text { Time, in days, post stroke to } \\
\text { randomisation }\end{array}$ & TBC & 118 \\
\hline $\begin{array}{l}\text { Location of stroke } \\
\text { Left MCA - 1 }\end{array}$ & Left MCA - 1 \\
\hline $\begin{array}{l}\text { Side of hemiparesis }(n) \text { L/R } \\
\text { of medians }{ }^{*}\end{array}$ & Right MCA - 3 & Right MCA - 3 \\
\hline $\begin{array}{l}\text { Wolf Motor Function Test - Functional } \\
\text { Ability (Mean of means }{ }^{*}\end{array}$ & Left ACA - 1 & Right Ventral Pons - 1 \\
\hline $\begin{array}{l}\text { Motor Activity Log - Amount of Use } \\
\text { (Mean) max=5 }\end{array}$ & 3.8 & $4 / 1$ \\
\hline
\end{tabular}




\begin{tabular}{|l|l|l|}
\hline $\begin{array}{l}\text { Motor Activity Log - Quality of Use (Mean) } \\
\text { max=5 }\end{array}$ & 2.2 & 2.0 \\
\hline Grip strength - Dynamometer (Mean) & 11.5 & 4.5 \\
\hline Mobility (indoors) & $\begin{array}{l}2 \text { independent, 2 } \\
\text { independent with walking } \\
\text { aid, 1 wheelchair }\end{array}$ & $\begin{array}{l}2 \text { independent, 3 } \\
\text { independent with walking } \\
\text { aid }\end{array}$ \\
\hline $\begin{array}{l}\text { Participants who had carers assisting }(n) \\
\text { Yes/No }\end{array}$ & $4 / 1$ & $3 / 2$ \\
\hline Drinking and smoking & $\begin{array}{l}2 \text { ex-smokers, 2 non- } \\
\text { smokers, 1 current } \\
\text { smoker }\end{array}$ & $\begin{array}{l}1 \text { ex-smoker, 4 non- } \\
\text { smokers }\end{array}$ \\
\hline
\end{tabular}

*The usual summary method for WMFT is described in the Methods section.

Figure 1. GRASP consort flow diagram.

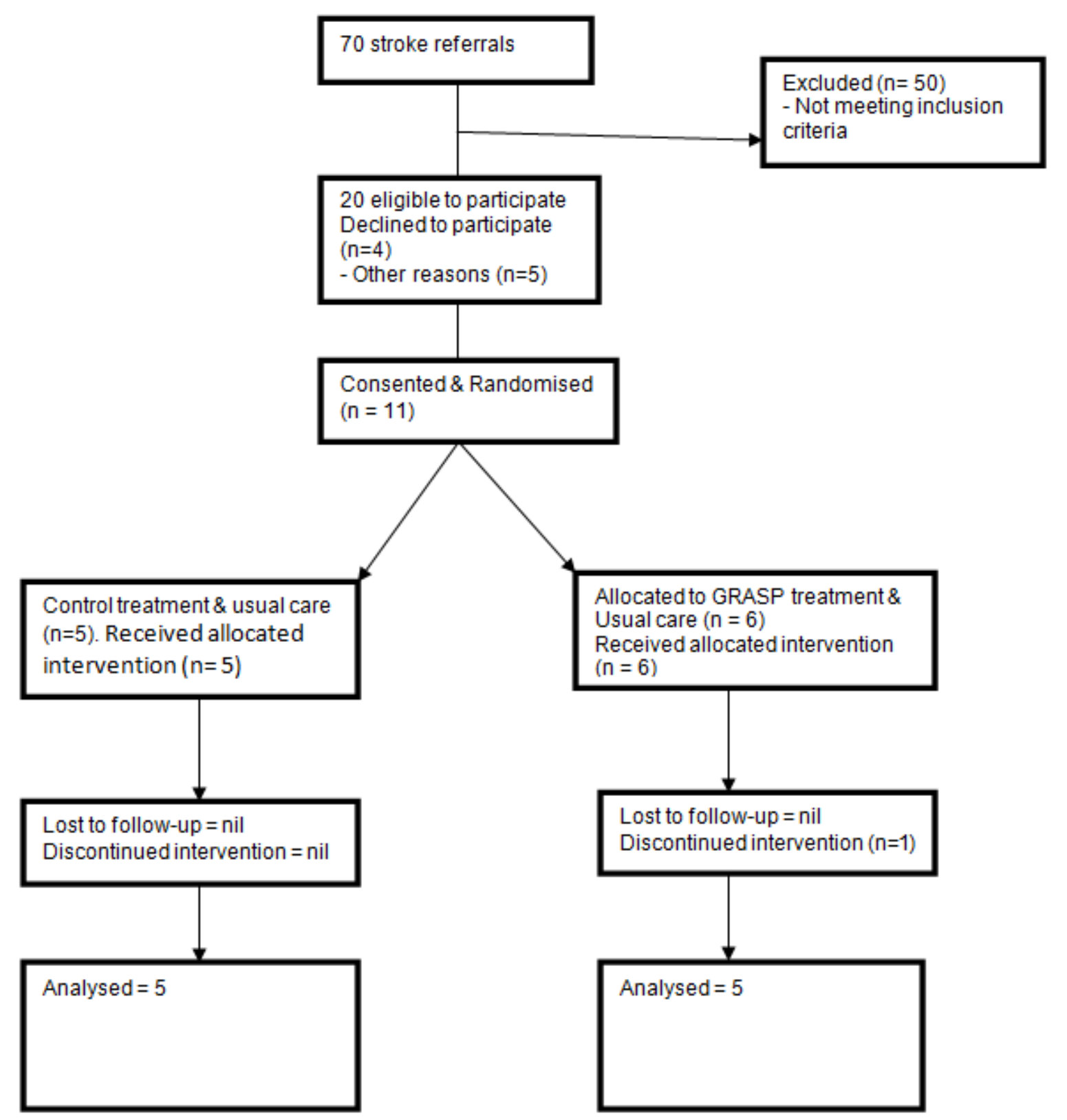


Intervention

The intervention was the GRASP programme that has been manualized and is available for download (Eng et al, 2012).

The components of GRASP are exercises that incorporate: range of motion and stretching; functional strengthening; weight-bearing through the hand; trunk control; repetitive paretic arm practice; repetitive bilateral arm tasks. Equipment is recommended, for example light weights, clothes pegs and paper clips. Eligibility criteria are outlined and details of the individual sessions are detailed in the GRASP manual (Eng et al. 2012).

Participants' family members were encouraged to be present during therapy sessions, should they wish (Jones and Riazi, 2011). Therapists upgraded or downgraded GRASP exercises according to participants' performance and their clinical. The control group received standard therapy and therapists were allowed to prescribe any other home exercise programme but not the GRASP protocol, reflecting standard practice for home-based upper limb rehabilitation after stroke. Irrespective of group allocation, each participant was advised to practise exercises at least 1 hour a day, 5 days a week.

\section{Outcomes}

The Wolf Motor Function Test (WMFT), which consists of 15 tasks (Box 1) suggest and two strength measures, was used as the primary outcome measure, because it captures both impairment and disability (Wolf et al, 2001, 2005). The validity, reliability and responsiveness of theWMFT has been tested with both subacute and chronic stroke participants (Morris et al, 2001; Lin et al, 2009; Wu et al, 2011a, 2011b). We followed the detailed WMFT written protocols (Taub et al, 2011). The participants completed various single- and multiple-joint tasks in a timed manner, and movement quality was assessed by the investigator, using an ordinal functional ability scale. In all WMFT tasks, participants who did not complete a task within 120 seconds, or declined to attempt the task, were recorded as ' 120 '. Thus, the times are a mixture of a binary outcome (completed or not) and a time outcome, which is right-censored (a data point is above a certain value but it is unknown by how much) in statistical terminology.

Secondary outcome measures were the Motor Activity Log and dynamometer grip strength. The Motor Activity Log is widely used in clinical research and is a semi-structured interview where participants respond with a number corresponding to a given amount of use and quality of movement during activities of daily living (van der Lee et al, 2004). The 14-item Motor Activity Log version was used and it is validi and reliable with post-stroke participants in real-world upper limb use (Uswatte et al, 2005). It is internally consistent, highly stable and responsive for people with mild to moderate upper limb hemiparesis. The mean of three attempts with a Jamar dynamometer was used to measure grip strength. Reliability and validity has been well established for the handheld dynamometer for stroke patients (Mathiowetz, 2002; Bertrand et al, 2007; Harris et al, 2009).

All outcome measures were taken at the participant's home at baseline and at follow-up after 4 weeks. Time spent both in standard therapy and GRASP/other supplemented therapy, along with pain, fatigue and assistance received, was recorded by both groups in log sheets. At weekly visits, therapists checked completion of these log sheets, but they were availed to the investigators after the outcomes. Part of the reason for the log sheets was also for weekly safety monitoring by the treating therapists, but also to provide a self-reported participation record for the two groups.

Box 1. Wolf Motor Function Test tasks

All tasks are performed as quickly as possible and are truncated at 120 seconds. Tasks are as 
follows: 1 . Forearm to table (side): Subject attempts to place forearm on the table by abduction at the shoulder.

2. Forearm to box (side): Subject attempts to place a forearm on the box by abduction at the shoulder.

3. Extend elbow (side): Subject attempts to reach across the table by extending the elbow (to the side).

4. Extend elbow (to the side), with weight: Subject attempts to push the sandbag against outer wrist joint across the table by extending the elbow.

5. Hand to table (front): Subject attempts to place involved hand on the table.

6. Hand to box (front): Subject attempts to place hand on the box.

7. Reach and retrieve (front): Subject attempts to pull $1 \mathrm{lb}$ weight across the table by using elbow flexion and cupped wrist.

8. Lift can (front): Subject attempts to lift can and bring it close to lips with a cylindrical grasp.

9. Lift pencil (front): Subject attempts to pick up pencil by using 3-jaw chuck grasp.

10. Pick up paper clip (front): Subject attempts to pick up paper clip by using a pincer grasp.

11. Stack checkers (front): Subject attempts to stack checkers onto the center checker.

12. Flip cards (front): Using the pincer grasp, patient attempts to flip each card over.

13. Turning the key in lock (front): Using pincer grasp, while maintaining contact, patient turns key fully to the left and right.

14. Fold towel (front): Subject grasps towel, folds it lengthwise, and then uses the tested hand to fold the towel in half again.

15. Lift basket (standing): Subject picks up basket by grasping the handles and placing it on bedside table

\section{Sample size and power}

Although this study investigated feasibility with a convenience sample of five participants in each group, a power calculation showed that this would have $80 \%$ power to detect a difference of 10 seconds on a WMFT performance time, assuming a standard deviation of 5.9 seconds and a significance set at $P<0.05$. This number was increased to seven per group, to allow for a predicted drop-out from treatment of around $30 \%$.

\section{Statistical analysis}

The Mann-Whitney test was used to compare the GRASP and control arms in terms of each individual WMFT task's time (taking '120' seconds at face value), each task's functional ability scale score, total Motor Activity Log, and average grip strength. We used this non-parametric test because of the small sample size, asymmetry of outcome data about the mean and lack of information on what distribution of values might be expected in the population, given that GRASP has rarely been evaluated in this setting.

As suggested by Wolf et al (2001), we calculated the median time taken across all WMFT tasks for each participant, and the mean functional ability scale score and then compared these by Mann-Whitney test. However, we were concerned at loss of information in this approach and developed our own alternative, described in more detail below. These statistical tests were performed using R statistical software (R-Development Core Team, 2014).

In order to combine all information across all tasks and outcomes, we used a Bayesian structural equation model (Figure 2), which was estimated using WinBUGS software (Lunn et al, 2000). There are three major advantages to this approach:

- Participants who could not complete a task and are recorded with a time of ' 120 ' seconds can be included alongside the others by using a binary outcome as to whether they could complete the task; this is akin to a survival analysis with censored survival times, rather than taking ' 120 ' at face value 
- Individual participants and individual tasks are given estimated 'impairment' and 'difficulty' scores, in the manner of an item-response theory model; these are useful in comparing the model to reality

- All 15 tasks' time and functional ability score, along with grip strength, could be incorporated into one model that pools all the data to show a difference in underlying impairment, rather than subdividing them into 31 separate tests, each of which relies on very few observations and is likely to be so uncertain that no conclusion can be drawn.

Bayesian methods are often regarded as synonymous with subjective opinions, but this is not necessary, and in this case, we did not take that approach, using diffuse prior distributions instead. (Lunn et al; 2000; Song and Lee, 2012). Further information, including the code and data, are available https://github.com/robertgrant/grasp.

The WinBUGS software, with a burn-in of 1000 iterations to achieve convergence was used, then evaluated over 200000 iterations thinned to every tenth to reduce autocorrelation.

Diffuse conjugate priors were used throughout. Times taken in each task were logarithmically transformed to an approximate normal distribution (after adding one second to avoid very large negative values), and those that were not completed were incorporated by regarding the times as a mixture of two normal distributions: one containing the log-times to completion and the other containing the incomplete tasks with a shared large mean and very small standard deviation. To achieve this we replaced the 120 s with values of 900 , sufficiently far removed from the completed times to clearly be from a different distribution. As the completion of the task was known, this allowed participants who could not complete a task before treatment, but could afterwards, to 'switch' from one distribution to the other without artificially large changes in WMFT time unduly dominating the data.

The patients' impairment and tasks' difficulty are represented as crossed random effects. So, although this model included all results on all tasks at both time points, it does not regard these as independent observations but as arising from a specific patient and a specific task.

Figure 2. Structural equation model. Rectangles indicate data; Ellipses indicate latent variables; Regression parameters are estimated for each of the arrows. FAS: Functional ability scale.

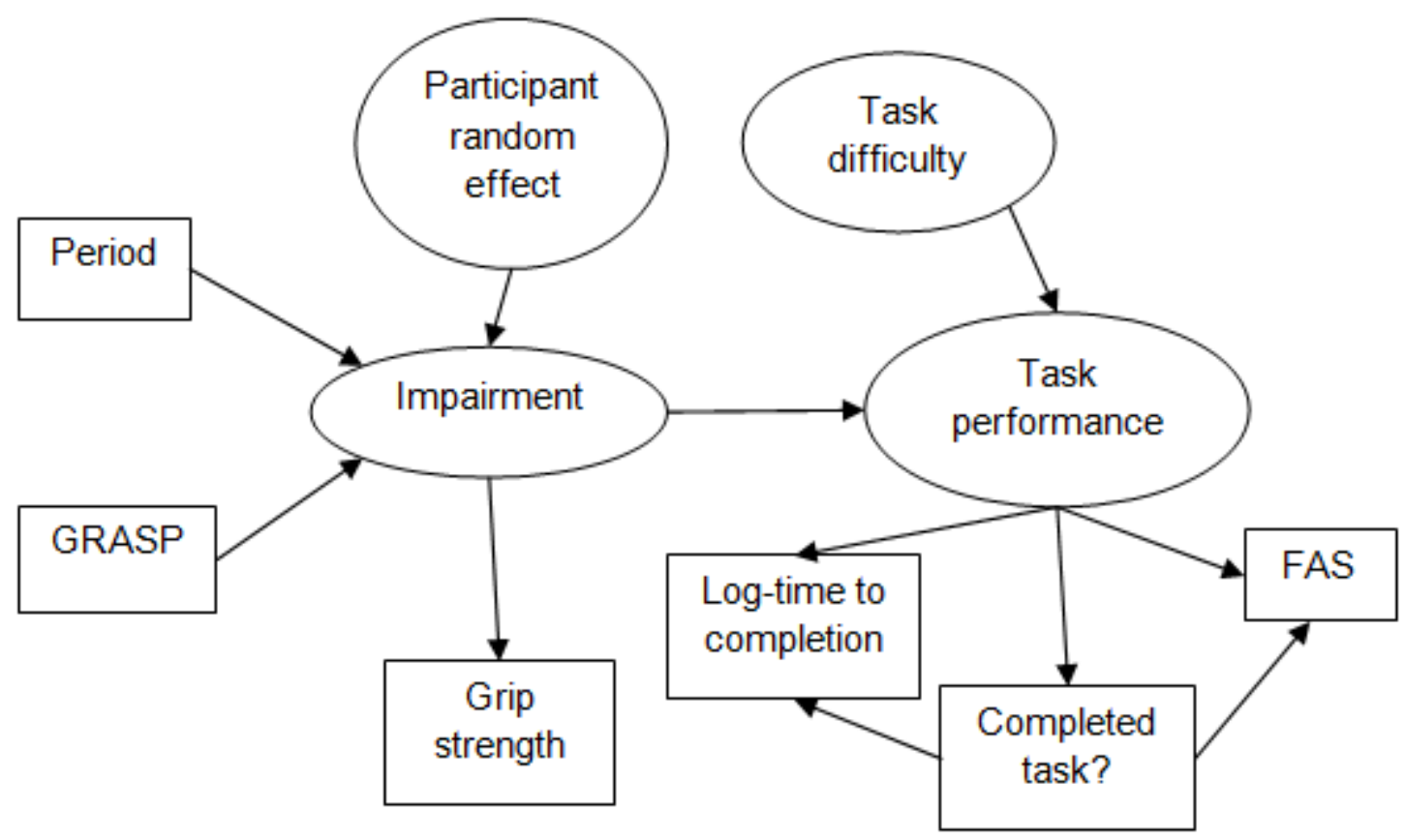


Using log-transformed time as an outcome means that the effect of GRASP is measured on a multiplicative scale as a relative reduction in time to completion, which is realistic given the greater potential for improvement in participants who were more impaired at baseline.

\section{Results}

At baseline, the two groups were similar in characteristics as shown in Table 1, although the GRASP group had faster WMFT performance, assessed by Wolf's median of medians (discussed in the Statistical analysis section).

In the control arm, participants received an average of 11.2 hours of conventional rehabilitation with a therapist, and supplemented this with 4.8 hours in their own time $(43 \%$ supplement). In the GRASP group, participants received an average of 10 hours' rehabilitation with the therapist and supplemented this with an average of 16 hours in their own time $(160 \%$ supplement).

There were no serious side effects reported by either group. Two participants, one from each treatment group, reported painful wrists and hands which subsided after the first week. One participant in the control group reported fatigue, and one participant in the GRASP group reported shoulder pain; in both cases these symptoms subsided after the first week.

\section{Estimate of effect}

Wolf et al (2001) recommend using the median time improvement and the mean functional ability scale improvement for analysis. The median improvement in time taken in each WMFT task was greater in the GRASP group than the control group for 12/15 tasks, and the remaining three tasks had the same improvement in both groups. However, only task number 2 (forearm to box, $P=0.04$ ) and task number 11 (pick up paper clip, $P=0.03$ ) were statistically significant at the $5 \%$ level using Mann-Whitney tests $(n=10)$. Before the intervention, five tasks had three participants in the control group recorded as '120', which artificially fixes the median time at 120 (tasks 3,4,5,7,10 of the WMFT). After the intervention period, three tasks had a majority of participants recorded as 120 .

The mean improvement in functional ability scale scores was greater in the GRASP group for $13 / 15$ of the tasks, with one equal between groups and one favouring control. Only task number 5 (hand to table, $P=0.02$ ) and number 6 (hand to box, $P=0.02$ ) were statistically significant by the Mann-Whitney test $(n=10)$, both favouring the GRASP group. These comparisons for individual tasks are shown in more detail in Figure 3.

Figure 3. Comparison of results in 15 WMFT tasks, and in summary. Data are only shown for participants and tasks where the task was completed both pre- and post-treatment. The graph is truncated to data between -20 and +20 seconds, purely for clarity reasons because the overall change is close to zero in comparison. Four observations deteriorated by more than 20 seconds, all in the control group. Three observations improved by more than 20 seconds, two in the GRASP group and one in the control group. The overall effect diamonds are from the structural equation model (Figure 2). 


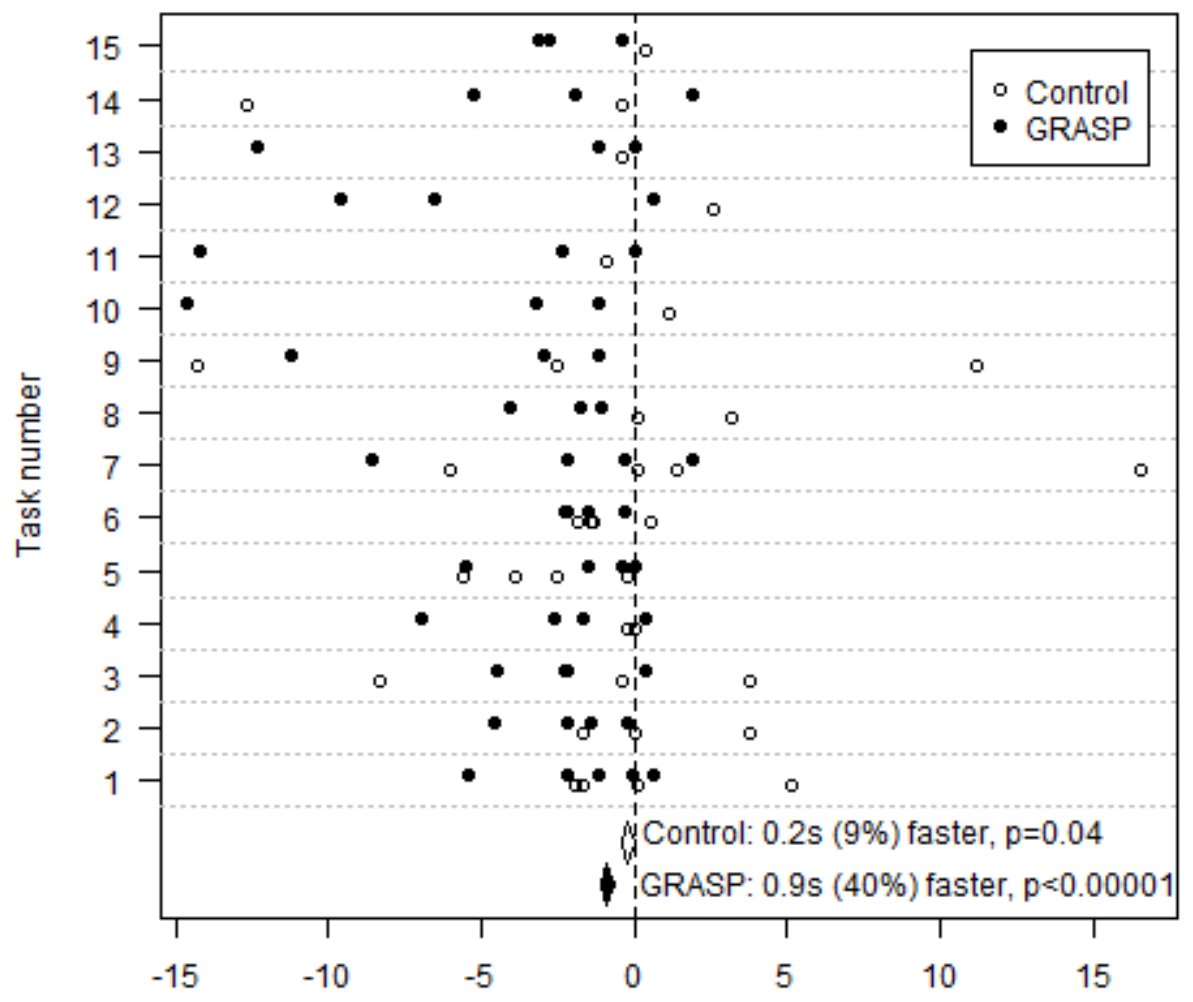

Change in time to completion (seconds: negative=faster)

The recommended summary measures for WMFT, the median time taken and the mean functional ability scale, were respectively not significant $(P=0.21)$ and significant $(P=0.04)$ by the Mann-Whitney test, both favouring the GRASP group. Median improvements in quality of movement, amount of use and grip strength were all greater in the GRASP group but none was significantly different between the treatment arms.

These individual tasks mostly favoured the GRASP group, but the potential for falsely significant results among the many Mann-Whitney tests, and the fact that even the significant results were close to $P=0.05$, which is characteristic of chance findings in multiple testing, led us to be wary of drawing conclusions from them. Instead, we focused on combining the data into one statistical assessment in the structural equation model (Figure 2). This provided strong evidence for an overall benefit of GRASP on latent impairment, as manifest in the log-transformed time to completion ( $P<0.001$, time to task completion was reduced by $40 \%$ in the GRASP group, $95 \%$ confidence interval 30-49\%), odds of non-completion ( $P<0.001$, odds ratio $=0.05$ implying that for every 20 tasks not completed in the control group, only one was not completed after GRASP, 95\% confidence interval $0.02-0.14)$, and functional ability scale $(P<0.001$, mean difference $=0.54,95 \%$ confidence interval $0.39-0.68)$. The effect on grip strength was not significant $(P=0.77$, mean difference $=0.57,95 \%$ confidence interval $-3.75-5.21$ ).

\section{Discussion}

One of the main tenets of treatment outcome in rehabilitation is to promote independence, and this study's results confirmed previous findings that GRASP may promote independence through improved upper limb function (Pang et al, 2006). This study, although small, has demonstrated the feasibility of implementing GRASP in a community setting and also indicates possible benefit within one month. GRASP was successfully implemented and delivered in a community setting and there is potential for further research using GRASP in the community. At a 
time when there is pressure on stroke rehabilitation resources, innovative approaches to community rehabilitation should be promoted, including research on their efficacy.

It is already a known fact that a higher dosage of therapy leads to better outcomes (Lohse et al. 2014).. However, in the current economic environment, it is difficult to sustain current levels of therapy within the UK NHS, let alone more therapy visits to patients in the community. A key positive finding was the additional hours of practice undertaken by the GRASP group. This study has shown that GRASP may be a consideration as therapists attempt to address the question of how to provide more therapy to community-dwelling stroke patients.

\section{Delivering more therapy}

GRASP does not involve the usual one-to-one client: therapist ratio, which is characteristic of other rehabilitation approaches such as constrained induced movement therapy ((Pang et al, 2006). This is potentially an innovative way of delivering more therapy and with limited health budgets/resources, an innovative community rehabilitation approach is needed. This feasibility study has shown there are potentially better rehabilitation outcomes using fewer resources.

The GRASP programme allowed participants to supplement their therapy: $160 \%$ versus $43 \%$ from the control group. This marked increase supports previous findings that GRASP adds critical upper limb therapy practice (Harris et al, 2009). The importance of face-to-face interaction with the therapist in delivering GRASP is uncertain as the study of phone monitoring saw target activity in 5 out of 6 participants and superiority on one mode of delivery over the other needs to be tested [(Simpson et al, 2017). Monitoring and contact seems important, and requires further exploration to ascertain clinical and cost effectiveness of this approach. The selection criteria for GRASP are as not stringent as alternative treatments such as constrained induced movement therapy (Wolf et al, 2006; Myint et al, 2008; Harris et al, 2009). GRASP is innovative as it has three different levels that cater for different levels of motor/functional difficulties, making it easier for a therapists to tailor the exercise progression.

There was no intervention contamination between the groups as all participants were seen in their home environment, so control subjects will not have been able to access the GRASP protocol. Although the study raised the general awareness of GRASP as a treatment method, therapists did not change their treatment of participants in the control group as confirmed through study monitoring and clinical supervision provided to therapists.

\section{Data analysis}

Because of the sample size (10 participants $\times 2$ time points), individual WMFT tasks showed either small non-significant differences between arms, or significant differences in favour of GRASP. Some statistical power was also lost by the use of non-parametric tests. By pooling the data across all WMFT tasks and investigating completion, time to completion, functional ability scale and grip strength in a unified structural equation model (10 participants $\times 2$ time points $\times 15$ tasks $\times 3$ measures, plus grip strength in 10 participants $\times 2$ time points), we were able to test the overall hypothesis that GRASP affects underlying impairment, which in turn affects the various outcome measures.

The data analysis demonstrates that conducting statistical tests on individual tasks in the WMFT may lack statistical power. Because GRASP affects impairment and all parts of WMFT assess aspects of impairment, it makes sense to combine the data into one global comparison of GRASP and control. Further, the recommended WMFT summary measures also discard a lot of information from individual participants and individual tasks. Consequently, studies may fail to detect true differences, or may require excessive sample sizes to achieve adequate statistical power. We recommend that, to make full use of WMFT data, researchers use the structural equation model approach, which can be implemented in standard Bayesian analysis software such as Bayesian inference Using Gibbs Sampling (BUGS), Just Another Gibbs Sampler (JAGS) or Stan (Plummer, 2003; Lunn et al, 2014; Stan Development Team, 2014). 
This study was limited as it had a small sample size, did not involve any follow-up after posttreatment measures nor did it collect participants' or caregivers' perceptions of the treatment received. In addition, participation in rehabilitation in both treatment arms was monitored using the self-reported log. The accuracy of completion was not established so there may have been a tendency to over-report engagement in rehabilitation.

However, given that GRASP in the community is feasible, and that promising results were obtained from this small sample of patients, a larger trial should be conducted to establish the scale of benefit, dose-response and barriers to adherence. Since long-term-therapy is now being delivered in the community, there is scope for studying how to optimise integration and handover from hospital to community. To the best of our knowledge, although GRASP is now widely known and used, this is the first independent study on GRASP and GRASP delivered by therapists in the community setting.

\section{Conclusions}

In this small, heterogeneous sample, the results suggest that GRASP may give greater improvement in both physical and functional recovery than conventional treatment control. These findings will add to the cumulative knowledge and inform future studies about GRASP and is not intended to be conclusive. A large randomised controlled trial on GRASP in the community is therefore recommended. As a self-management option, GRASP can be used to increase the dosage of critical therapy delivered to the upper limb after stroke in the community. GRASP was shown to be safe, feasible and has potential to be cost-effective in the treatment of the upper limb post-stroke for this group of participants used in a community setting.

\section{Acknowledgements}

We acknowledge the support of Central London Community Healthcare NHS Trust and its rehabilitation teams and St George's, University of London. We thank our participants and therapists, without whose tireless and diligent effort this study could not have been completed.

\section{Conflicts of interest}

The authors declare no conflicts of interest.

\section{Funding}

This work was completed as part of a Masters in Clinical Research programme funded by the National Institute for Health Research.

\section{References}

Alawieh A, Zhao J, Feng W. Factors affecting post-stroke motor recovery: Implications on neurotherapy after brain injury. Behav Brain Res. 2018;340:94-101. doi:10.1016/j.bbr.2016.08.029

Barreca S, Wolf SL, Fasoli S, Bohannon R. Treatment interventions for the paretic upper limb of stroke survivors: a critical review. Neurorehabil Neural Repair. 2003;17(4):220-226. https://doi.org/10.1177/0888439003259415

Bernhardt J, Chitravas N, Meslo IL, Thrift AG, Indredavik B. Not all stroke units are the same: a comparison of physical activity patterns in Melbourne, Australia, and Trondheim, Norway. Stroke. 2008;39(7):2059-2065. https://doi.org/10.1161/STROKEAHA.107.507160 
Bertrand AM, Mercier C, Bourbonnais D, Desrosiers J, Gravel D. Reliability of maximal static strength measurements of the arms in subjects with hemiparesis. Clin Rehabil. 2007;21(3):248-257. https://doi.org/10.1177/0269215506070792

Connell LA, McMahon NE, Harris JE, Watkins CL, Eng JJ. A formative evaluation of the implementation of an upper limb stroke rehabilitation intervention in clinical practice: a qualitative interview study. Implement Sci. 2014;9:90. https://doi.org/10.1186/s13012-014-0090-3

Eng J, Harris J, Dawson A, Miller B. Graded repetitive arm supplementary program: a home-work based program to improve arm and hand function in people living with stroke. Guidelines and Manual. 2012. http://neurorehab.med.ubc.ca/files/2012/10/GRASP-Instruction-Manual_2012.pdf (accessed 22 May 2020)

Folstein MF, Folstein SE, McHugh PR. Mini-mental state. A practical method for grading the cognitive state of patients for the clinician. J Psychiatr Res. 1975;12(3):189-198.

https://doi.org/10.1016/0022-3956(75)90026-6

Harris JE, Eng JJ, Miller WC, Dawson AS. A self-administered Graded Repetitive Arm Supplementary Program (GRASP) improves arm function during inpatient stroke rehabilitation: a multi-site randomized controlled trial. Stroke. 2009;40(6):2123-2128.

https://doi.org/10.1161/STROKEAHA.108.544585

Jones F, Riazi A. Self-efficacy and self-management after stroke: a systematic review. Disabil Rehabil. 2011;33(10):797-810. https://doi.org/10.3109/09638288.2010.511415

Khan CM, Oesch PR, Gamper UN, Kool JP, Beer S. Potential effectiveness of three different treatment approaches to improve minimal to moderate arm and hand function after stroke - a pilot randomized clinical trial. Clin Rehabil. 2011;25(11):1032-1041.

https://doi.org/10.1177/0269215511399795

Kollen BJ, Lennon S, Lyons B et al. The effectiveness of the Bobath concept in stroke rehabilitation: what is the evidence? Stroke. 2009;40(4):e89-e97.

https://doi.org/10.1161/STROKEAHA.108.533828

Langhorne P, Bernhardt J, Kwakkel G. Stroke rehabilitation. Lancet. 2011;377(9778):1693-1702. https://doi.org/10.1016/S0140-6736(11)60325-5

Lin K, Hsieh Y, Wu C et al. Minimal detectable change and clinically important difference of the Wolf Motor Function Test in stroke patients. Neurorehabil Neural Repair. 2009;23(5):429-434. https://doi.org/10.1177/1545968308331144

Lohse KR, Lang CE, Boyd LA. Is more better? Using metadata to explore dose-response relationships in stroke rehabilitation. Stroke. 2014;45(7):2053-2058.

doi:10.1161/STROKEAHA.114.004695

Lunn D, Thomas A, Best N. WinBUGS, a Bayesian modelling framework: concepts, structure and extensibility. Stat Comput. 2000;10:325-337. https://doi.org/10.1023/A:1008929526011

Mathiowetz V. Comparison of Rolyan and Jamar dynamometers for measuring grip strength. Occup Ther Int. 2002;9(3):201-209. https://doi.org/10.1002/oti.165

Morris DM, Uswatte G, Crago JE, Cook EW, Taub E. The reliability of the wolf motor function test for assessing upper extremity function after stroke. Arch Phys Med Rehabil. 2001;82(6):750-755. https://doi.org/10.1053/apmr.2001.23183 
Myint JMWW, Yuen GFC, Yu TKK et al. A study of constraint-induced movement therapy in subacute stroke patients in Hong Kong. Clin Rehabil. 2008;22:112-124.

https://doi.org/10.1177/0269215507080141

Pang MY, Harris JE, Eng JJ. A community-based upper-extremity group exercise program improves motor function and performance of functional activities in chronic stroke: a randomized controlled trial. Arch Phys Med Rehabil. 2006;87(1):1-9. https://doi.org/10.1016/j.apmr.2005.08.113

Plummer M. JAGS: A program for analysis of Bayesian graphical models using Gibbs sampling. 2003. https://www.r-project.org/conferences/DSC-2003/Proceedings/Plummer.pdf (accessed 4 June 2020)

R-Development Core Team. R: a language and environment for statistical computing. 2014. http://www.gbif.org/resource/81287 (accessed 22 May 2020)

Saka O, McGuire A, Wolfe C. Cost of stroke in the United Kingdom. Age Ageing. 2009;38(1):27-32. https://doi.org/10.1093/ageing/afn281

Simpson LA, Eng JJ, Chan M. H-GRASP: the feasibility of an upper limb home exercise program monitored by phone for individuals post stroke. Disabil Rehabil. 2017;39(9):874-882.

https://doi.org/10.3109/09638288.2016.1162853

Song XY, Lee SY. Basic and advanced Bayesian structural equation modeling: with applications in the medical and behavioral sciences. Chichester: John Wiley and Sons; 2012

Stan Development Team. StanStan modeling language users guide and reference manual. 2014. http://mc-stan.org/ (accessed 22 May 2020)

Stroke Association. State of the nation: stroke statistics. 2018.

https://www.stroke.org.uk/sites/default/files/state of the nation 2018.pdf (accessed 21July2020)

Taub E, Morris D, Crago J, Wolf motor function test manual. 2011.

https://www.uab.edu/citherapy/images/pdf_files/CIT_Training_WMFT_Manual.pdf (accessed 22 May 2020)

Uswatte G, Taub E, Morris D, Vignolo M, McCulloch K. Reliability and validity of the upper-extremity Motor Activity Log-14 for measuring real-world arm use. Stroke. 2005;36(11):2493-2496.

https://doi.org/10.1161/01.STR.0000185928.90848.2e

van der Lee JH, Beckerman H, Knol DL, de Vet HCW, Bouter LM. Clinimetric properties of the motor activity log for the assessment of arm use in hemiparetic patients. Stroke. 2004;35(6):14101414. https://doi.org/10.1161/01.STR.0000126900.24964.7e

Ward NS, Kelly K, Brander F. Intensive upper limb neurorehabilitation in chronic stroke: outcomes from the Queen Square programme. J Neurol Neurosurg Psychiatry. 2018;90(5):498-506.

https://doi.org/10.1136/jnnp-2018-319954

Ward NS, Kelly K, Brander F. An expert opinion: upper limb rehabilitation after stroke. ACNR. 2019;18(4):20-22. https://doi.org/10.3109/09638288.2013.824034

Wolf SL, Catlin PA, Ellis M et al. Assessing Wolf motor function test as outcome measure for research in patients after stroke. Stroke. 2001;32(7):1635-1639.

https://doi.org/10.1161/01.STR.32.7.1635 
Wolf SL, Thompson PA, Morris DM et al. The EXCITE trial: attributes of the Wolf Motor Function Test in patients with subacute stroke. Neurorehabil Neural Repair . 2005;19(3):194-205.

https://doi.org/10.1177/1545968305276663

Wolf SL, Winstein CJ, Miller JP et al. Effect of constraint-induced movement therapy on upper extremity function 3 to 9 months after stroke: the EXCITE randomized clinical trial. JAMA. 2006;296(17):2095-2104. https://doi.org/10.1001/jama.296.17.2095

Wu C, Chuang L, Lin K, Chen H, Tsay P. Randomized trial of distributed constraint-induced therapy versus bilateral arm training for the rehabilitation of upper-limb motor control and function after stroke. Neurorehabil Neural Repair. 2011a;25(2):130-139.

https://doi.org/10.1177/1545968310380686

Wu C, Fu T, Lin K et al. Assessing the streamlined Wolf motor function test as an outcome measure for stroke rehabilitation. Neurorehabil Neural Repair. 2011b;25(2):194-199.

https://doi.org/10.1177/1545968310381249

Young J, Forster A. Review of stroke rehabilitation. BMJ. 2007;334(7584):86-90.

https://doi.org/10.1136/bmj.39059.456794.68 